\title{
SMALL PERTURBATIONS AND THE EIGENVALUES OF THE LAPLACIAN ON LARGE BOUNDED DOMAINS
}

\author{
WERNER KIRSCH
}

\author{
(Communicated by John B. Conway)
}

\begin{abstract}
Denote by $\Delta_{L}^{D}$ the Laplacian on a hypercube in $\mathbf{R}^{d}$ with side length $\pi L$. Also denote by $N(\lambda, A)$ the number of eigenvalues of the operator $A$ below $\lambda$. If $V \geq 0$ is a bounded function of compact support, $(V>0$ on a set of positive measure $)$ then $N\left(-\Delta_{L}^{D}, \lambda\right)-N\left(-\Delta_{L}^{D}+V, \lambda\right)$ is not bounded as $L \rightarrow \infty$ for dimension $d>1$.
\end{abstract}

1. Introduction. Let $C_{L}$ be the hypercube in $\mathbf{R}^{d}$ for $d>1$ with side length $\pi L$ centered at the origin. Denote by $\Delta_{L}^{D}$ the Laplacian on $C_{L}$ with Dirichlet boundary conditions on $\partial C_{L}$. Let $V$ be a bounded nonnegative potential with support in $C_{1}$ and set $H_{0}^{(L)}=-\Delta_{L}^{D}, H_{1}^{(L)}=-\Delta_{L}^{D}+V$.

If $H$ is a selfadjoint operator bounded below with purely discrete spectrum we denote by $E_{n}(H), n=0,1, \ldots$, the eigenvalues of $H$ put in increasing order and counted according to their multiplicity. We set $N(H, \lambda)=\#\left\{n \mid E_{n}(H) \leq \lambda\right\}$. The main object of investigation in this note is the function

$$
\varphi_{L}(\lambda)=N\left(H_{0}^{(L)}, \lambda\right)-N\left(H_{1}^{(L)}, \lambda\right) .
$$

For any $L \varphi_{L}(\lambda)$ is a nonnegative function.

We ask whether $\varphi_{L}(\lambda)$ remains bounded for fixed $\lambda$ if $L$ goes to infinity. Our intuition (and the one of many experts I asked) suggests that $\varphi_{L}$ should be bounded since the perturbation $V$ is independent of $L$ and so the "effect of $V$ " should not grow with $L$ but should rather become smaller. However, this intuition is erroneous, as we shall prove below.

As a corollary we obtain that also

$$
\tilde{\varphi}_{L}(\lambda)=N\left(-\Delta_{C_{L}-C_{1}}^{D, N}\right)-N\left(-\Delta_{C_{L}-C_{1}}^{D, D}\right)
$$

is unbounded where $-\Delta_{C_{L}-C_{1}}^{D, N}$ (resp. $-\Delta_{C_{L}-C_{1}}^{D, D}$ ) is $-\Delta$ on $L^{2}\left(C_{L}-C_{1}\right.$ ) with Dirichlet boundary conditions on $\partial C_{L}$ and Neumann (resp. Dirichlet) boundary conditions on $\partial C_{1}$. The unboundedness of $\tilde{\varphi}_{L}$ is perhaps even less intuitive than that of $\varphi_{L}$.

Our proof that $\varphi_{L}$ is unbounded as $L$ increases relies heavily (though not obviously) on the high symmetry of the Laplacian, as was pointed out to us by H. Siedentop. It would be interesting to know the behavior of $\varphi_{L}$ for $-\Delta$ replaced by some $H=-\Delta+W$. However, our method does not work in that case.

It is a pleasure to thank V. Enss, F. Gesztesy, and H. Siedentop for clarifying discussions.

Received by the editors May 27, 1986 and, in revised form August 11, 1986 and October 7, 1986.

1980 Mathematics Subject Classification (1985 Revision). Primary 35P20, 35J10, 47 A70.

Key words and phrases. Dirichlet Laplacian, small perturbation, eigenvalue distribution. 
2. The result. We are ready to state our result. Assume that $V(x)>0$ on a set of positive Lebesgue measure.

THEOREM 1. (i) For any $\lambda>0$ we have

$$
\sup _{L \in \mathbf{R}} \varphi_{L}(\lambda)=\infty .
$$

(ii) For a dense set $E \subset[0, \infty)$ and any $\lambda \in E$ we have

$$
\sup _{L \in \mathbf{N}} \varphi_{L}(\lambda)=\infty \text {. }
$$

3. Proof of (i). To prove this result we consider first the eigenvalues of the Laplacian $-\Delta_{L}^{D}$ on $C_{L}$. We denote by $\mathbf{Z}_{++}$the strictly positive integers. For $\mathbf{n} \in\left(\mathbf{Z}_{++}\right)^{d}$ we set $|\mathbf{n}|=\left(\sum_{i=1}^{d} n_{i}^{2}\right)^{1 / 2}$. The eigenvalues of $-\Delta_{L}^{D}$ are given by

$$
E_{n}^{(L)}=\frac{1}{L^{2}} \sum_{i=1}^{d} n_{i}^{2} \quad \text { for } \mathbf{n} \in\left(\mathbf{Z}_{++}\right)^{d} .
$$

The multiplicity of $E_{\mathbf{n}}^{(L)}$ equals the number of points $\mathbf{m} \in\left(\mathbf{Z}_{++}\right)^{d}$ with $|\mathbf{m}|=|\mathbf{n}|$ (see Reed-Simon [2]). The first observation is

Proposition 1. For any $M$ there is an $\mathbf{n} \in\left(\mathbf{Z}_{++}\right)^{d}$ such that $|\mathbf{m}|=|\mathbf{n}|$ has at least $M$ distinct solutions $\mathbf{m} \in\left(\mathbf{Z}_{++}\right)^{d}$.

PROOF. It is enough to prove the result for $d=2$. In this case the number $l(a), a \in \mathbf{N}$, of solutions $m \in \mathbf{Z}^{2}$ of $|m|^{2}=a$ is exactly known by the following theorem of Gauss.

THEOREM (GAUSS). If

$$
a=2^{n^{n}} p_{1}^{n_{1}} p_{2}^{n_{2}} \cdots p_{r}^{n_{r}} q_{1}^{m_{1}} q_{2}^{m_{2}} \cdots q_{s}^{m_{s}}
$$

is the decomposition of a into prime factors with $p_{i} \equiv 1 \bmod 4$ and $q_{i} \equiv 3 \bmod 4$, the number $l(a)$ of distinct solutions of $|m|^{2}=a$ with $m \in \mathbf{Z}^{2}$ is given by

$$
l(a)= \begin{cases}4\left(n_{1}+1\right)\left(n_{2}+1\right) \cdots\left(n_{r}+1\right) & \text { if } m_{i}=0 \bmod 2 \text { for all } i, \\ 0 & \text { otherwise }\end{cases}
$$

For a proof we refer to Fricker [1, Satz 1 in §2].

From this theorem the assertion of the proposition follows.

COROLlaRY. For any $M, L_{0}, E_{0}>0$ there is an $L>L_{0}$ such that $E_{0}$ is an eigenvalue of $H_{0}^{(L)}$ of multiplicity at least $M$.

PROOF. By Proposition 1 there is a number $\alpha>E_{0} L_{0}^{2}$ such that $|\mathbf{m}|^{2}=\alpha$ has at least $M$ solutions $\mathbf{m} \in\left(Z_{++}\right)^{d}$. So with $L:=\left(\alpha / E_{0}\right)^{1 / 2}>L_{0}$ we have

$$
E_{0}=\frac{1}{L^{2}} E_{\mathbf{m}}^{(1)}=E_{\mathbf{m}}^{(L)} \text {. }
$$

Moreover $E_{\mathbf{m}}^{(L)}$ has multiplicity at least $M$.

The idea of the proof is that all the eigenvalues of $H_{1}^{(L)}$ are strictly bigger than the corresponding eigenvalues of $H_{0}^{(L)}$. So, if $\lambda=E_{\mathbf{m}}^{(L)}$ has multiplicity at least $M$ the function satisfies $\varphi_{L}(\lambda) \geq M$. 
Thus the proof of (i) is done once we know

PROPOSITION 2. For any $L$ and $n \in \mathbf{N}$

$$
E_{n}\left(H_{1}^{(L)}\right)>E_{n}\left(H_{0}^{(L)}\right) .
$$

ProOF. Suppose that $f_{0}, \ldots, f_{m}$ is an orthonormal set of eigenfunctions of $H_{0}^{(L)}$ spanning Rank $P_{\left(-\infty, E_{n}\left(H_{0}^{(L)}\right)\right)}$ where $P$ denotes the spectral projection of $H_{1}^{(L)}$. Denote by $D$ the common domain of the operators $H_{0}^{(L)}$ and $H_{1}^{(L)}$. By the min-max-principle (see Reed-Simon [2]) we have

$$
\begin{aligned}
E_{n}\left(H_{0}^{(L)}\right) & =\inf _{\substack{\varphi \in D,|\varphi|=1 \\
\varphi \perp f_{0}, \ldots, f_{m}}}\left\langle\varphi, H_{0}^{(L)} \varphi\right\rangle \\
& \leq \inf _{\substack{\varphi \in D,|\varphi|=1 \\
\varphi \perp f_{0}, \ldots, f_{m}}}\left\langle\varphi, H_{1}^{(L)} \varphi\right\rangle \leq E_{n}\left(H_{1}^{(L)}\right)
\end{aligned}
$$

It is a standard compactness argument to show that the infimum

$$
\inf _{\substack{\varphi \in D,|\varphi|=1 \\ \varphi \perp f_{0}, \ldots, f_{m}}}\left\langle\varphi, H_{1}^{(L)} \varphi\right\rangle
$$

is attained by some function $\varphi_{0} \in D,|\varphi|=1$.

Now, suppose that equality holds in $(*)$. Then

$$
\left\langle\varphi_{0}, H_{1}^{(L)} \varphi_{0}\right\rangle=\left\langle\varphi_{0}, H_{0}^{(L)} \varphi_{0}\right\rangle=\inf _{\substack{\varphi \in D,|\varphi|=1 \\ \varphi \perp f_{0}, \ldots, f_{m}}}\left\langle\varphi, H_{0}^{(L)} \varphi\right\rangle
$$

and consequently $\varphi_{0}$ is an eigenfunction of $H_{0}^{(L)}$ to the eigenvalue $E_{n}\left(H_{0}^{(L)}\right)$, as can be checked by the min-max-principle. Thus $\left|\varphi_{0}\right|^{2}$ is strictly positive outside a set of Lebesgue measure zero. Therefore $\int\left|\varphi_{0}(x)\right|^{2} V(x)>0$. But this contradicts $\left\langle\varphi_{0}, H_{0}^{(L)} \varphi_{0}\right\rangle=\left\langle\varphi_{0}, H_{1}^{(L)} \varphi_{0}\right\rangle$, thus proving the proposition.

REMARK. The poof of Proposition 2 applies to more general situations than described above.

4. The proof of (ii). Again it suffices to prove the statement for $d=2$. Set $A=\left\{n \in \mathbf{N} \mid n=a^{2}+b^{2}\right.$ for $\left.a, b \in \mathbf{Z}_{++}\right\}$and $\tilde{A}=\{p / q \mid p, q \in A\}$.

Proposition 3. If $\lambda \in \tilde{A}$ then $\sup _{L \in \mathbf{N}} \varphi_{L}(\lambda)=\infty$.

ProOF. Suppose $\lambda=p / q$ with $p, q \in A$, then

$$
\lambda=\frac{1}{5^{2 k} q^{2}} 5^{2 k} q p=\frac{1}{L^{2}} \alpha
$$

with $L=5^{k} q$ and $\alpha=5^{2 k} q p$. Using Gauss' theorem we easily see that $\alpha \in A$, so that $\lambda$ is an eigenvalue of $H_{0}^{(L)}$. Again by Gauss' theorem the multiplicity of this eigenvalue increases to infinity as $L \rightarrow \infty$. Thus, by Proposition 2 , we have $\lim _{k \rightarrow \infty} \varphi_{5^{k} q}(\lambda)=\infty$.

It remains to show

Proposition 4. $\tilde{A}$ is dense in $[0, \infty)$. 
To see this we will make use of the following lemma:

LemMA. For any $p \in \mathbf{Z}_{++}$there exists an $m \in A$ such that $p \leq m \leq p+$ $2(p / 2)^{1 / 2}+1$.

PROOF. If $n=a^{2}+b^{2}$ with $a \leq b$ then $a \leq(n / 2)^{1 / 2}$; define $n^{\prime}=(a+1)^{2}+b^{2}=$ $n+2 a+1$. Then

$$
n^{\prime}-n=2 a+1 \leq 2(n / 2)^{1 / 2}+1 .
$$

Now given $p>2$ set $n_{p}=\max \{m \in A \mid m \leq p\}$ then by definition $n_{p}^{\prime}>p$. Moreover

$$
n^{\prime} \leq n+2(n / 2)^{1 / 2}+1 \leq p+2(p / 2)^{1 / 2}+1,
$$

which proves the lemma.

We are prepared to prove Proposition 4:

PROOF. First we prove that any integer $r>0$ can be approximated by certain $r_{n} \in \tilde{A}$. Indeed, the Lemma tells us that any of the intervals $I_{n}=\left[2^{n} r, 2^{n} r+\right.$ $2\left(2^{n-1} r\right)^{1 / 2}+1$ ] has nonempty intersection with $A$. Denote the smallest element of $A$ within $I_{n}$ by $m_{n}$ and set $r_{n}=m_{n} / 2^{n}$. Then $r_{n} \in \tilde{A}$ and

$$
\left|r_{n}-r\right|=m_{n} / 2^{n}-r=\frac{1}{2^{n}}\left(m_{n}-2^{n} r\right) \leq \frac{1}{2^{n}}\left(2\left(2^{n-1} r\right)^{1 / 2}+1\right) \rightarrow 0 .
$$

Now let $r=p / q$ be an arbitrary positive rational number. By multiplying, if necessary, $p$ and $q$ by the same number, we may assume without loss of generality that $q \in A$. By the above considerations we have $p_{n} \in \tilde{A}$ approaching $p$ as $n \rightarrow \infty$. Set $r_{n}=p_{n} / q$; then $r_{n}$ belongs to $\tilde{A}$. Moreover $r_{n}=p_{n} / q \rightarrow p / q=r$.

5. A consequence. Let us denote by $\Delta_{C_{L}-C_{1}}^{D, N}$ the Laplacian on $C_{L}-C_{1}$ with Dirichlet conditions at the outer boundary, i.e. at $\partial C_{L}$, and with Neumann condition at the inner boundary, i.e. at $\partial C_{1}$. By $\Delta_{C_{L}-C_{1}}^{D, D}$ we denote the Laplacian on the same region with Dirichlet conditions on the whole boundary. As above one might argue that

$$
\tilde{\varphi}_{L}(\lambda)=N\left(-\Delta_{C_{L}-C_{1}}^{D, N}, \lambda\right)-N\left(-\Delta_{C_{L}-C_{1}}^{D, D}, \lambda\right)
$$

should be bounded, since the surface where the boundary condition is changed remains the same for all $L$. However, the same assertions as in Theorem 1 are valid for $\varphi_{L}$ replaced by $\tilde{\varphi}_{L}$. The reason is a simple Dirichlet-Neumann bracketing argument (see Reed-Simon [2]):

$$
\begin{aligned}
\varphi_{L}(\lambda) & =N\left(-\Delta_{C_{L}}^{D}, \lambda\right)-N\left(-\Delta_{C_{L}}^{D}+V, \lambda\right) \\
& \leq N\left(-\Delta_{C_{L}-C_{1}}^{D, N}+-\Delta_{C_{1}}^{N}, \lambda\right)-N\left(-\Delta_{C_{L}-C_{1}}^{D, D}+\left(-\Delta_{C_{1}}^{D}+V\right), \lambda\right) \\
& =N\left(-\Delta_{C_{L}-C_{1}}^{D, N}, \lambda\right)-N\left(-\Delta_{C_{L}-C_{1}}^{D, D}, \lambda\right)+N\left(-\Delta_{C_{1}}^{N}, \lambda\right)-N\left(-\Delta_{C_{1}}^{D}+V, \lambda\right) \\
& \leq \tilde{\varphi}_{L}(\lambda)+\left(N\left(-\Delta_{C_{1}}^{N}, \lambda\right)-N\left(-\Delta_{C_{1}}^{D}, \lambda\right)\right) .
\end{aligned}
$$

Since the difference above is independent of $L$ the result follows.

\section{REFERENCES}

1. F. Fricker, Einführung in die Gitterpunktlehre, Birkhäuser, 1982.

2. M. Reed and B. Simon, Methods of modern mathematical physics, Vol. IV, Academic Press, 1978. 